\title{
HIERARCHICAL BLIND WATERMARKING OF 3D TRIANGULAR MESHES
}

\author{
Kai Wang , Guillaume Lavoué*, Florence Denis ${ }^{* *}$ and Atilla Baskurt ${ }^{*}$ \\ *Laboratoire LIRIS, UMR 5205 CNRS, INSA-Lyon, Villeurbanne, F-69621 France \\ ** Laboratoire LIRIS, UMR 5205 CNRS, Université Lyon 1, Villeurbanne, F-69622 France
}

\begin{abstract}
An original hierarchical watermarking scheme is proposed in this paper. A geometrically robust watermark and a high-capacity watermark are inserted in different resolution levels of the wavelet decomposition of a semi-regular mesh by modifying the norms of wavelet coefficients. Both watermarks are blind and invariant to similarity transformations. The robustness of the first watermark is achieved by synchronizing and quantizing watermark primitives according to edges lengths of the coarsest level, which are quite insensible to geometrical attacks. The high capacity of the second watermark is obtained by considering the permutation of the norms of a group of wavelet coefficients. Experiments have proven the high robustness of the first watermark under common geometrical attacks. To our knowledge, the capacity of the second method, which is equal to the factorial of the candidate coefficients number, is the highest for $3 \mathrm{D}$ meshes in the literature.
\end{abstract}

\section{INTRODUCTION}

Digital watermarking has been considered as a potential efficient solution for copyright protection of various multimedia contents. This technique carefully hides some secret information in the cover content. Compared with traditional cryptography, digital watermarking technique is able to protect digital works after the transmission and the legal access.

In computer graphics, a 3D object is usually represented by a polygon mesh, which is a collection of polygonal facets targeting to approximate the real shape of the 3D object. With the increasing capability of capturing, processing and visualizing 3D data, the protection of 3D meshes has attracted more and more attention. However, there still exist few watermarking methods for 3D meshes, in contrast with the relative maturity of the theory and practices of image, audio and video watermarking. This situation is mainly caused by the difficulties encountered while handling the arbitrary topology and irregular sampling of 3D meshes, and the complex possible geometrical and topological attacks on watermarked meshes.

Existing techniques concerning 3D meshes can be classified into two main categories, depending whether the watermark is embedded in the spatial domain (by modifying the geometry or the connectivity) or in the spectral domain (by modifying some kind of spectral coefficients). Most early methods fall in the first category, authors attempted to insert watermarks in different spatial primitives. These primitives include the distance from a vertex to the gravity centre of the mesh [13], the average normal direction of a group of facets [1], the projection of a vertex on its opposite edge [4], the ratio between triangle height and opposite edge length [9], the local triangulation density [9], the relative position of a vertex to its 1-ring neighbors [3], and so on. Usually, this kind of methods is sensitive to connectivity attacks, such as simplification and remeshing. To overcome this drawback, some algorithms propose a resampling stage on the input mesh of the watermark extraction to recover the same connectivity as the original cover mesh, but this inevitably makes the method non-blind. The second category of algorithms firstly decomposes the mesh in a transformed spectral domain, and then the watermark is inserted whether at the low, median or high frequency part [6, 10-12]. These methods usually provide better imperceptibility and better robustness to geometrical attacks, whereas the connectivity issue is still a problem. For example, the Laplacian spectral analysis of 3D meshes is sensible to connectivity change [10]; the edge decimation technique to establish a multiresolution representation is also dependant to the mesh connectivity [11]; and most wavelet analysis tools require the input as semi-regular mesh, in which most vertices are of valence six [6].

In this paper, an original hierarchical watermarking scheme is proposed for semi-regular meshes. A robust watermark and a highcapacity watermark co-exist in the mesh for copyright protection and content labeling purposes, respectively. The remainder of this paper is organized as follows. Section 2 presents the principles of wavelet analysis of 3D meshes and the overview of our global blind hierarchical watermarking method for semi-regular meshes. In section 3, the insertion and extraction of the geometrically robust blind watermark is described. Section 4 introduces a watermark whose capacity increases rapidly with the number of insertion primitives. The proposed hierarchical scheme is validated by some experimental results in section 5 . Finally, we conclude and point out several future working directions in section 6 .

\section{OVERVIEW OF THE PROPOSED FRAMEWORK}

The objective is to build a robust and blind 3D mesh watermarking scheme. First of all, the cover mesh (possibly irregular) is remeshed to generate a corresponding semi-regular mesh with a similar geometrical shape. Then multiple watermarks are inserted in this semi-regular mesh. For extraction, we suppose that a mesh with the same semi-regular connectivity can be reconstructed. Here, the connectivity issue is supposed to be solved. The key point lies in elaborating a remeshing scheme which is insensitive to connectivity change. So the research on hierarchical watermarking of semi-regular meshes in this paper can be considered as a basic block of this framework and doesn't lose generality. Furthermore, a semi-regular mesh normally has a negligible geometrical distortion compared to the original one and is more favorable for compression thanks to its simple connectivity [7].

The watermarking algorithm for semi-regular meshes presented in this paper is based on wavelet analysis. The 
mathematical formulation of the wavelet analysis and synthesis of 3D meshes was introduced by Lounsbery [8]. Such an analysis provides a coarse mesh which represents the basic shape (low frequencies) and a set of wavelet coefficients which stands for details information at each resolution level (median and high frequencies). Figure 1 illustrates one iteration of the lazy wavelet decomposition mechanism. A group of four triangles is merged in one and three of the six initial vertices are conserved in the lower resolution. The wavelet coefficients are calculated as the prediction errors for all the deleted vertices and they are 3D vectors associated with each edge of the coarser mesh. One straightforward prediction is the midpoint of two conserved vertices having been incident to the deleted vertex. Note that this kind of wavelet analysis is applicable only on semi-regular triangular meshes having 4-1 subdivision connectivity. In our algorithms, the watermarks are inserted by modifying the coefficients norms.

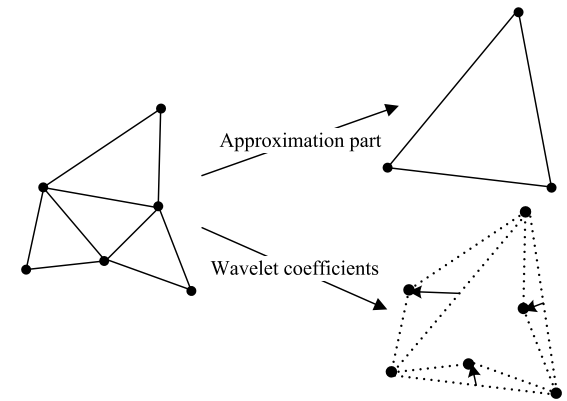

Fig. 1. Illustration of 3D triangular mesh wavelet analysis

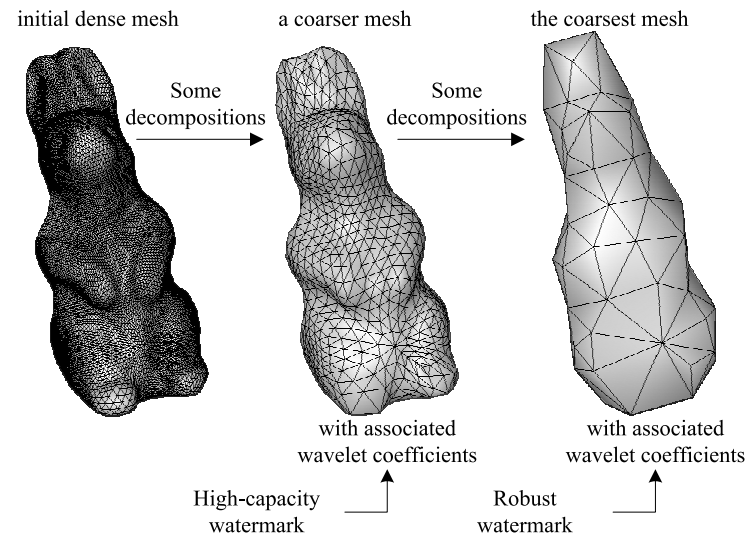

Fig. 2. Hierarchical watermarking of semi-regular mesh

Multiresolution analysis is a very suitable tool for hierarchical watermarking since there is no inter-infection between different watermarks if they are inserted in different levels. In our method, the robust watermark is in the coarsest level ensuring copyright protection, and the high-capacity watermark is in a denser level according to the expected number of bits to hide (capacity), as illustrated in figure 2 . Since the connectivity attacks are assumed to be solved by the remeshing step, it is sufficient for the watermarks in the semi-regular mesh to be robust to geometrical attacks.

\section{ROBUST AND BLIND WATERMARKING}

One critical problem of blind watermarking schemes is their relatively weak robustness. Generally speaking, the synchronizing mechanism is often more fragile than the real watermark modulation scheme. In fact, during the extraction, it is difficult to successfully find out the locations and indexes of the watermark bits. In the case of 3D meshes, the situation is even worse, because there isn't any robust ordering for the basic elements of the mesh (vertices, edges and facets), which often constitute the watermarking primitives. Our proposal is to utilize some robust aspect to locate and index the embedded bits. Practically, the sort order of the edges in the coarsest level, established on their lengths, is experimentally very robust to geometrical attacks. The watermark bits are inserted one by one in the wavelet coefficients norms associated with the ordered edges. Moreover, in this way, the synchronizing mechanism and the watermarking primitives are separated, so the causality problem is avoided.

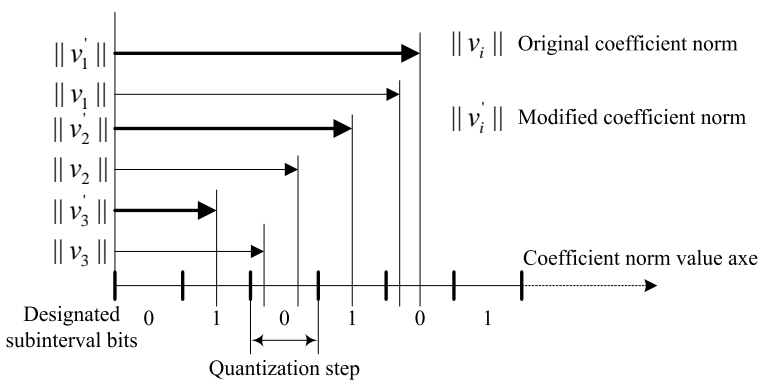

Fig. 3. Three quantization examples of coefficient norms

The watermarking scheme of the wavelet coefficients norms is quite simple. The real number axe is divided into subintervals by a quantization step, and each subinterval is designated to associate with a certain bit ( 0 or 1$)$. This designation could be determined by a secret key, but here we simply adopt the interleaving bit string of 0 and 1. All the norms are altered to locate in the middle of the nearest correct subinterval according to the subsequent bit to be inserted, while the coefficients directions are kept unchanged. Figure 3 shows three quantization examples: we want to encode " 0 11 " in the coefficients associated with three longest edges, $\left\|v_{1}\right\|$ is already in the correct subinterval, so it is moved to the middle of this subinterval; $\left\|v_{2}\right\|$ and $\left\|v_{3}\right\|$ have to be displaced to the middle of the nearest correct subinterval in order to introduce less distortion.

The quantization step is determined by the average length of the edges mentioned before. In fact, in this method, the real watermarking primitive is the ratio between the norm of a coefficient and the average length of all the edges in the coarsest level. This kind of primitive is proven to be invariant to similarity transformations including translation, rotation and uniform scaling. Algorithm 1 presents the whole watermark insertion flow. $\varepsilon_{1}$ is a control parameter to achieve an expected trade-off between robustness and imperceptibility. If the edges number is greater than the watermark bits number, a redundant embedding will be done to enhance the robustness. Knowing the parameter $\varepsilon_{1}$, which can also be hard-cored in the program, the extraction is blind and quite simple. It is sufficient to reestablish the edges order, calculate the quantization step, and find out the designated bit of each coefficient under this quantization step.

\footnotetext{
Algorithm 1: Robust and blind watermarking method

1. Do wavelet analysis until the coarsest level

2. Do descending sort of all the edges in this level by their lengths

3. Calculate the average length $l_{\mathrm{av}}$ of the edges and fix the quantization step as $l_{\mathrm{av}} / \varepsilon_{1}$
} 
Repeat for each edge in the descending sort

4. Calculate the norm of its associated wavelet coefficient

5. Quantize this norm according to the next watermark bit value using the above mechanism

End repeat

6. Reconstruct the densest semi-regular mesh with the modified wavelet coefficients

\section{HIGH-CAPACITY BLIND WATERMARKING}

Under some circumstances, a high-capacity watermark is needed, to carry more information such as the description of a multimedia file, the patient information of a CT scan, or the screen information of a digital photo. In the literature, the highest capacity for 3D meshes is nearly one bit per triangle $[2,9]$. In this section, a new scheme with a much higher capacity is introduced. In this scheme, the watermark is no longer inserted bit by bit, but globally.

For a given mesh at a certain level of wavelet decomposition, we suppose that its $n$ edges are sorted by their lengths, as in the last section. Then the sort of the $n$ wavelet coefficients norms associated with these ordered edges has $n$ ! possibilities. In fact, each possibility can be modeled as a permutation of the $n$ numbers ranging from 1 to $n$ and represents a watermark of floor $\left(\log _{2} n\right.$ !) bits, where $f l o o r(x)$ is a function that returns the largest integer less than or equal to $x$ (so practically some permutations have no corresponding watermarks). It seems natural to modify this original order of the norms into a particular one to insert a designated watermark. Actually, to achieve a better imperceptibility, we sort and modify the residues of the norms modulo a control parameter $p$, instead of the norms themselves. Same to the robust watermark, this control parameter is fixed to $p=l_{a v} / \varepsilon_{2}$ and is also determined by the average length of the edges (but at a different level). The modified norm is determined by equation (1), where $\operatorname{order}(i)$ is the new permutation order of the coefficient that is associated with $i^{\text {th }}$ edge. Table 1 gives the watermarking procedure for a simple example of 4 edges, where $p=1$. The correspondence between permutations and watermark bit strings will be explained later on.

$$
\left\|v_{i}^{\prime}\right\|=\text { floor }\left(\frac{\left\|v_{i}\right\|}{p}\right) * p+\frac{\operatorname{order}(i) * p}{(n+1)}
$$

Table 1. Example of high-capacity watermark embedding steps

\begin{tabular}{|c|c|c|c|c|}
\hline Edges lengths & 12 & 10 & 9.2 & 8.5 \\
\hline Edges order $(i)$ & 1 & 2 & 3 & 4 \\
\hline $\begin{array}{c}\text { Associated wavelet } \\
\text { coefficients norms }\end{array}$ & 2.3 & 1.5 & 1.8 & 2.1 \\
\hline Residues of norms modulo $p$ & 0.3 & 0.5 & 0.8 & 0.1 \\
\hline Original coefficients order & 2 & 3 & 4 & 1 \\
\hline Expected order $($ order $(i))$ & 1 & 4 & 3 & 2 \\
\hline Modified residues & 0.2 & 0.8 & 0.6 & 0.4 \\
\hline Modified norms & 2.2 & 1.8 & 1.6 & 2.4 \\
\hline
\end{tabular}

In practice, the $n$ edges can be divided into several groups of $m$ edges (in each group are inserted floor $\left(\log _{2} m\right.$ !) bits) in order to make the watermark less fragile and to avoid the possible floating number calculation errors. So the practical capacity of this method is floor $(n / m) *$ floor $\left(\log _{2} m\right.$ !) bits. Figure 4 graphically compares the capacities of different high-capacity methods of the state of the art: Cayre's method (1 bit/vertex) [4], Ohbuchi's method $(\approx 1.2$ bits/triangle) [9], Benedens' method (1 bit/triangle, not presented in the figure, almost superposes the curve of the Ohbuchi's method) [2], and ours ( $m=n$ and $m=30)$. It is assumed that for a manifold triangular mesh, we usually have $e=1.5 f$ and $f \approx 2 v$, where $v, e$ and $f$ are the numbers of vertices, edges and facets of the mesh, respectively.

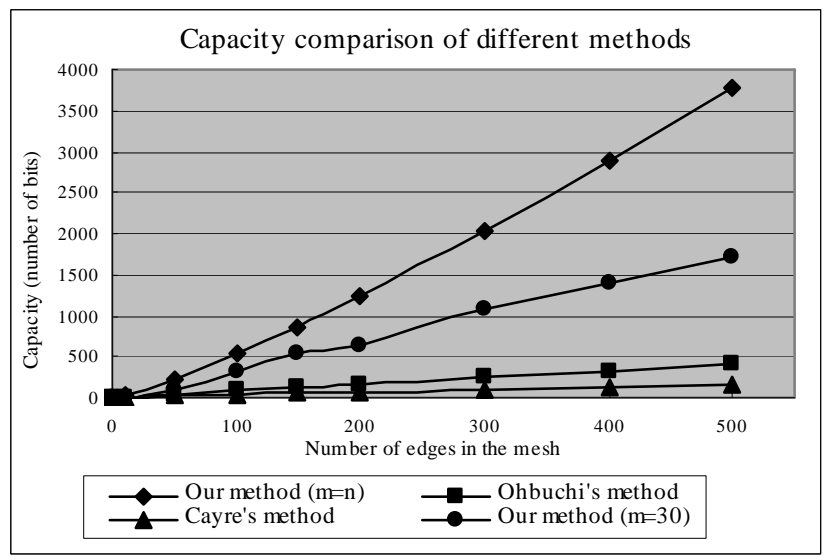

Fig. 4. Capacity comparison of different methods

A correspondence between watermarks (bit strings) and possible norms orders (permutations) has to be established. The basic rule adopted is that for two permutations, the one with a bigger first number (from left) represents a bigger bit string. And if the first number is the same, we compare the second, and so on. Under this rule, the permutation $1,2,3, \ldots, n-1, n$ represents the smallest bit string $0,0, \ldots, 0,0$, and the permutation $1,2,3, \ldots, n$, $n$ - 1 designates the bit string $0,0, \ldots, 0,1$.

If the control parameter $p$ here is equal to the quantization step in section 3, the norm distortion in the worst situation is almost the same for these two watermarking algorithms. However, the high-capacity watermark relies on the relationship between norms of different coefficients, so even a little norm attack smaller than $p$ could seriously disrupt the original order. That is the principal reason for the relative fragility of this watermark.

\section{EXPERIMENTAL RESULTS}

We have tested our hierarchical algorithm on different objects. The robust watermark has to be inserted before the high-capacity watermark so as not to disturb the edges order used by the latter. The execution time varies along with the complexity of the input semi-regular mesh. For the rabbit mesh illustrated in Figure 5.a having about $70 \mathrm{~K}$ vertices, the whole insertion procedure takes less than 10 seconds on a computer with a $1.7 \mathrm{GHz}$ Intel $^{\circledR}$ Pentium $^{\circledR}$ mobile processor and $512 \mathrm{MB}$ RAM. The semi-regular meshes are obtained by the algorithm proposed in [5].

Figure 5.b shows the watermarked rabbit mesh with zooms at the nose and neck. The parameter $\varepsilon_{1}$ is fixed in the experiments at 35 , and a robust watermark of 64 bits is repeatedly inserted with chip rate $c=3$ in the coarsest level (five decompositions from the initial dense mesh) which has 207 edges. The high-capacity watermark is inserted at level 3 which has 3312 edges. Taking $m=$ 30 , the real capacity is about $10.6 \mathrm{~K}$ bits. The parameter $\varepsilon_{2}$ is fixed at 40 . There are no visible geometrical distortions between these two meshes, and their mean distance, calculated on the two meshes centered and scaled in unit cube, is quite small $\left(D_{L 1}=\right.$ 0.003013 ).

To validate the robust watermarking scheme, we have attacked the watermarked rabbit mesh by random additive noises, average smoothing, enhancement, and vertices coordinate 
quantization operations. Figure 6 illustrates four attacked watermarked meshes, and Table 2 presents the experimental results, where the robustness is measured by bit error rate (BER) and correlation between the extracted watermark and the inserted one. The intensities of the additive noises are relative to the average length from vertices to the gravity centre. We can conclude that the robust watermark well resist to majority of the common geometrical attacks. The high-capacity watermark is invariant to similarity transformations, but is fragile to strong geometrical operations. The main reason is given at the end of section 4 . However, if inserted in the coarsest level, it can survive until $0.04 \%$ additive noise with a capacity of about 500 bits.

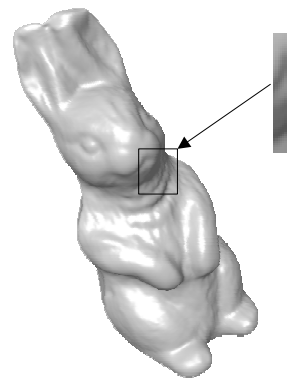

(a)

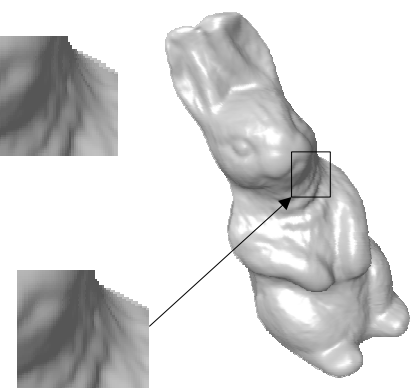

(b)
Fig. 5. Hierarchical watermarking example: (a) original semiregular rabbit mesh; (b) watermarked rabbit mesh.

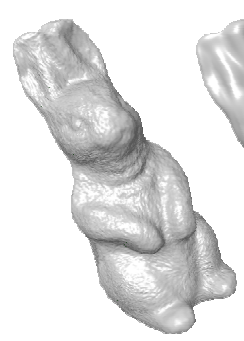

(a)

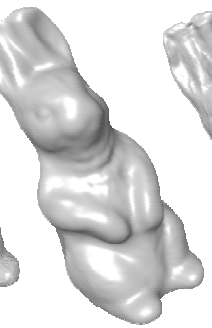

(b)

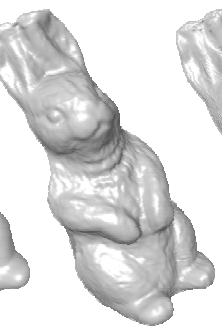

(c)

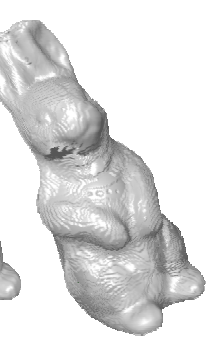

(d)
Fig. 6. Four attacked watermarked meshes: (a) $0.4 \%$ additive noise; (b) 6 average smoothing; (c) enhancement; (d) 7-bit quantization.

Table 2. Results of robustness experiments

\begin{tabular}{|c|c|c|c|}
\hline \multirow{2}{*}{ Attacks } & \multicolumn{2}{|c|}{ Robust watermark } & \multirow{2}{*}{$\begin{array}{l}\text { High-capacity } \\
\text { watermark }\end{array}$} \\
\hline & BER & Correlation & \\
\hline Similarity Trans. & 0 & 1 & Exist \\
\hline $0.04 \%$ noise & 0 & 1 & Exist* \\
\hline $0.25 \%$ noise & 0.046875 & $0.901319 * *$ & Lost \\
\hline $0.40 \%$ noise & 0.109375 & 0.769015 & Lost \\
\hline 6 smoothing & 0.03125 & 0.933333 & Lost \\
\hline Enhancement & 0.0625 & 0.866667 & Lost \\
\hline 8-bit quantization & 0.03125 & 0.933333 & Lost \\
\hline 7-bit quantization & 0.17875 & 0.630608 & Lost \\
\hline
\end{tabular}

* If inserted in the coarsest level; ** 0.92 in Yu's non-blind method [13].

\section{CONCLUSIONS AND FUTURE WORK}

In this paper, we have reported a new and effective blind hierarchical watermarking framework for semi-regular meshes which modifies the wavelet coefficients norms. Both watermarks are blind and invariant to similarity transformations. The use of robust aspects of the carrier to synchronize the watermark and the separation of the synchronization mechanism from watermark primitives is a good start point to elaborate robust and blind watermarking methods. Furthermore, although the high-capacity watermark is somewhat fragile, it is useful for steganography and content labeling. Future work includes improvement of the robust watermark to stand against local deformations and cropping. Studying remeshing techniques that are insensitive to connectivity change, improving the high-capacity watermark robustness, and taking account of mesh local geometrical properties for watermarking are also of interest.

\section{ACKNOWLEDGEMENTS}

The authors would acknowledge Céline Roudet for her help on wavelet analysis and synthesis of 3D meshes.

\section{REFERENCES}

[1]. O. Benedens, "Geometry-based Watermarking of 3D Models", IEEE Computer Graphics and Applications, vol. 19, no. 1, pp. 46-55, 1999.

[2]. O. Benedens, "Two High Capacity Methods for Embedding Public Watermarks into 3D Polygonal Models", Proceedings of the ACM Multimedia and Security Workshop, pp. 95-99, 1999.

[3]. A.G. Bors, "Watermarking Mesh-based Representations of 3D Objects Using Local Moments", IEEE Transactions on Image Processing, vol. 15, no. 3, pp. 687-701, 2006.

[4]. F. Cayre, and B. Macq, "Data Hiding on 3-D Triangle Meshes", IEEE Transactions on Signal Processing, vol. 51, no. 4, pp. 939-949, 2003.

[5]. I. Guskov, K. Vidimce, W. Sweldens, and P. Schroder, "Normal Meshes", Proceedings of the ACM SIGGRAPH Conference on Computer Graphics, pp. 95-102, 2000.

[6]. S. Kanai, H. Date, and T. Kishinami, "Digital Watermarking for 3D Polygons Using Multiresolution Wavelet Decomposition", International Workshop on Geometric Modeling, pp. 296-307, 1998.

[7]. A. Khodakovsky, P. Schroder, and W. Sweldens, "Progressive Geometry Compression", Proceedings of the ACM SIGGRAPH Conference on Computer Graphics, pp. 271-278, 2000.

[8]. M. Lounsbery, T.D. DeRose, and J. Warren, "Multiresolution Analysis for Surfaces of Arbitrary Topological Type", ACM Transactions on Graphics, vol. 16, no. 1, pp. 34-73, 1997.

[9]. R. Ohbuchi, H. Masuda, and M. Aono, "Data Embedding Algorithms for Geometrical and Non-geometrical Targets in Three-dimensional Polygonal Models", Computer Communications, vol. 21, no. 15, pp. 1344-1354, 1998.

[10]. R. Ohbuchi, A. Mukaiyama, and S. Takahashi, "A Frequency-domain Approach to Watermarking 3D Shapes", Computer Graphics Forum, vol. 21, no. 3, pp. 373-382, 2002.

[11]. E. Praun, H. Hoppe, and A. Finkelstein, "Robust Mesh Watermarking", Proceedings of the ACM SIGGRAPH Conference on Computer Graphics, pp. 49-56, 1999.

[12]. J. Wu, and L. Kobbelt, "Efficient Spectral Watermarking of Large Meshes with Orthogonal Basis Functions", Visual Computer, vol. 21, no. 8-10, pp. 848-857, 2005.

[13]. Z. Yu, H.H.S. Ip, and L.F. Kwok, "A Robust Watermarking Scheme for 3D Triangular Mesh Models", Pattern Recognition, vol. 36, no. 11, pp. 2603-2614, 2003. 